\title{
Comparison of fractionated frequency-doubled 1,064/532 nm picosecond Nd:YAG lasers and non-ablative fractional 1,540 nm Er: glass in the treatment of facial atrophic scars: a randomized, split-face, double-blind trial
}

\author{
Yu Shi ${ }^{1 \#}$, Wencai Jiang ${ }^{2 \#}$, Wei Li ${ }^{1 \#}$, Wei Zhang ${ }^{1}$, Ying Zou ${ }^{1}$ \\ ${ }^{1}$ Department of Medical Cosmetology, Tongji University Affiliated Shanghai Skin Disease Hospital, Shanghai, China; ${ }^{2}$ Department of Skin, and \\ Cosmetic Research Department, Tongji University Affiliated Shanghai Skin Disease Hospital, Shanghai, China \\ Contributions: (I) Conception and design: W Zhang, Y Shi; (II) Administrative support: Y Shi; (III) Provision of study materials or patients: Y Zou; \\ (IV) Collection and assembly of data: W Jiang, Y Shi; (V) Data analysis and interpretation: W Li; (VI) Manuscript writing: All authors; (VII) Final \\ approval of manuscript: All authors. \\ "These authors contributed equally to this work. \\ Correspondence to: Wei Zhang. Department of Medical Cosmetology, Tongji University Affiliated Skin Disease Hospital, 1278 Bao De Road, Shanghai \\ 200443, China. Email: Pflw111@126.com.
}

Background: Laser treatment of acne scars is common, but quality evidence on its efficacy is still needed. Our study aimed to compare picosecond laser and non-ablative fractional laser's efficacy and safety in treating acne atrophic scars.

Methods: This was a randomized, split-face double-blind trial recruiting patients with acne atrophic scars. Facial halves were randomly divided and treated with fractionated frequency-doubled 1,064/532 nm picosecond Nd:YAG laser or non-ablative fractional 1,540 nm Er: glass laser. ECCA score (echelle d'evaluation clinique des cicatrices d'acne) and skin flatness measured with a non-invasive phaseshift rapid in vivo measurement of skin (PRIMOS) system were evaluated one month after the last treatment.

Results: Twenty-two Fitzpatrick skin type IV patients were included in this study, with an average age of 29.68 years, an average duration of acne scars of 8.8 years. Picosecond laser impacted all acne scar types (before and after treatment; $\mathrm{P}=0.000$ for all types, $\mathrm{P}<0.001$ for $\mathrm{V}$-type, $\mathrm{P}=0.002$ for $\mathrm{U}$-type, and $\mathrm{P}=0.021$ for $\mathrm{M}$-type) and more pronounced effect on ECCA score than non-ablative laser for V-type and U-type acne scars. After treatment, each treatment site's height was significantly lower than that before treatment $(\mathrm{P}=0.041)$ in the picosecond group but not in the non-ablative group $(\mathrm{P}=0.785)$. The reported erythema rate was higher in patients treated with a picosecond laser, while edema, exudation, purpura, pain, and long-term AEs were similar between the groups.

Conclusions: Fractionated frequency-doubled 1,064/532 nm Picosecond Nd: YAG laser showed better efficacy in treating acne atrophic scars than the alternative and provided satisfactory safety with added improvement in pores and the glossiness of the skin.

Registration number: ChiCTR2100045982 (comparison of fractionated frequency-doubled 1,064/532 nm picosecond Nd: YAG lasers and nonablative fractional 1,540 nm Er: glass in the treatment of facial atrophic scar: a randomized, split-face, double-blind controlled trial).

Keywords: Acne scars; picosecond laser; randomized; split-face

Submitted Mar 22, 2021. Accepted for publication May 19, 2021.

doi: $10.21037 /$ atm-21-1715

View this article at: http://dx.doi.org/10.21037/atm-21-1715

(c) Annals of Translational Medicine. All rights reserved. 


\section{Introduction}

Acne is the most common chronic inflammatory disease of hair follicles and sebaceous glands in adolescents and adults. Studies showed that about $80 \%$ of people over 20 years old had ever suffered from acne $(1,2)$, and about $95 \%$ of acne patients would have acne scars of varying degrees (3). Facial atrophic scars can cause damage to the patient's appearance, often adversely affect the patient's social activities and mental health, and seriously affect the patient's quality of life $(4,5)$. Currently, acne scaring's main treatment methods include grinding, surgical release, plasma therapy, autologous fibroblasts, platelet-rich plasma, laser therapy (5), but many of these operations have reported flaws and are seriously limited by operator skill.

Laser therapy is currently the most widely used clinical treatment (2-5) for acne scarring. However, both traditional laser and lattice laser are from the photothermal effect principle, often difficult to control accurately, leading to damage to the surrounding normal tissues (6), and outcomes reported are often biased. Traditional lasers, including $\mathrm{CO}_{2}$ fractional lasers, have a beneficial effect on acne atrophic scars, but they can cause scabbing, lengthy work delay, obvious pain, and a higher risk of pigmentation after treatment (4). Therefore, finding a therapeutic method with sufficient efficacy and safety is the main challenge facing the clinic.

The holographic diffraction neodymiumdoped yttrium aluminum garnet (Nd: YAG) lattice picosecond laser is the lattice mode of picosecond laser (1), which is a non-ablative laser rejuvenation system. Using the laser-induced optical breakdown (LIOB) and laser-induced cavitation (LIC) techniques, the regeneration and remolding of collagen can be stimulated in the largest proportion, with the least damage to surrounding tissues, while the epidermis still is intact (7). Our preliminary study results showed the Nd: YAG lattice picosecond laser had sufficient efficacy in treating acne atrophic scar, with fewer side effects, short recovery period, mild pain, and high satisfaction.

High-quality evidence on using laser therapies, including picosecond lasers, is still lacking for people with acne scarring. No clinical research of picosecond laser treatment for acne scars in the Chinese population has currently been published. Thus, our study aimed to compare picosecond laser and non-ablative fractional laser's efficacy and safety in treating acne atrophic scars. We present the following article in accordance with the CONSORT reporting checklist (available at http://dx.doi.org/10.21037/atm-211715).

\section{Methods}

\section{Study design and patients}

Our study was randomized and split-face and included patients with acne atrophic scars of type III-IV skin from April to August 2020 in our hospital. Inclusion criteria: (I) type III-IV skin; (II) with mild to severe atrophic acne scars on the face; (III) stable condition for $>6$ months; (IV) signed informed consent and cooperated with the follow-up.

Patients with pregnancy, lactation, photosensitive skin disease, keloid constitution, sunburn hyperpigmentation, active lesions on facial skin, history of skin tumors, oral administration of retinoic acid drugs within six months, topical use of retinoic acid drugs, or receiving other treatments for acne scars within one year were excluded. The study was performed following the Declaration of Helsinki (as revised in 2013) principles and approved by the Ethics Committee of Tongji University Affiliated Shanghai Skin Disease Hospital (Approval number: 2020-20). All the patients signed written informed consent.

\section{Randomization}

We divided facial halves of all patients randomly into "Picosecond group" (treated with picosecond fractional 1,064/532 nm Nd: YAG laser) and "Non-ablative group" (treated with non-ablative fractional $1,540 \mathrm{~nm}$ Er: glass laser) by coin method.

\section{Treatment}

Topical anesthesia with $5 \%$ lidocaine cream was applied before laser treatment for every subject. A fractionated frequency-doubled 1,064/532 nm picosecond Nd: YAG system (PicoWay, Syneron-Candela Corporation, Wayland, MA), fitted with a holographic beam-splitting optic (PicoWay Resolve1, Syneron-Candela Corporation) was used to treat acne scars. The system delivers a $10 \times 10$ array of $150 \mathrm{~mm}$-diameter microbeams arranged in a $6 \mathrm{~mm} \times 6 \mathrm{~mm}$, square treatment area.

\section{The picosecond group}

Subjects received four monthly treatments with fractionated $1,064 \mathrm{~nm}$ or $532 \mathrm{~nm}$ wavelengths, using an Nd: YAG picosecond-domain laser incorporating a KTP, frequency doubling crystal fitted with the novel holographic beamsplitter optic. The laser delivered 1.9-2.9 mJ/microbeam at $1,064 \mathrm{~nm}$ with a pulse duration of $450 \mathrm{ps}$ and $0.2-$ 
$0.3 \mathrm{~mJ} / \mathrm{microbeam}$ at $532 \mathrm{~nm}$ with a pulse duration of $375 \mathrm{ps}$. The laser repetition rate was $10 \mathrm{~Hz}$.

Two laser passes were administered sequentially in rows at right angles, 4 passes vertical, and 4 passes horizontal, treating the affected areas by delivering pulses in a painting mode. The treating physician determined treatment energy on experience with the laser and tissue reaction, where erythema and mild petechiae were desired endpoints.

Energies for the 1,064 nm wavelength were 1.7-1.9 J/cm² for each treatment. For the $532 \mathrm{~nm}$ wavelengths, delivered energies were $0.2-0.22 \mathrm{~J} / \mathrm{cm}^{2}$ for each treatment. The energy was increased by $10-20 \%$ each time.

\section{The non-ablative group}

StarLux (Palomar) was used with an energy of $65-70 \mathrm{~J} / \mathrm{cm}^{2}$, scanning treatment was performed, each spot overlapped $10 \%$, and the treatment area was scanned four times.

As postoperative care, all patients were instructed to avoid sun exposure and apply either moisturizing cream or sunscreen on the treated areas of skin. Other topical products were not allowed during the protocol treatment and follow-up to obtain clean results.

\section{Primary endpoint}

The primary endpoint was the change of ECCA score at 1 month after the last treatment compared to the before treatment score. Two physicians (with 15 and 30 years of work experience, respectively), who were blinded to the grouping, evaluated the treatment efficacy. ECCA total score was calculated as $\mathrm{X}$ and Y's product, where $\mathrm{X}$ value reflected scar and was scored according to its shape, color, and course of the disease ( $\mathrm{V}$-type scar, 15 points; U-type scar, 20 points; M-type scar, 25 points). The $\mathrm{Y}$ value reflects the distribution and number of scars.

\section{Secondary endpoints}

Secondary endpoints included PRIMOS score and satisfaction. Non-invasive, rapid skin three-dimensional imaging system [PRIMOS, GFMesstechnik GmbH (GFM)] was used to measure each treatment site's height. The height map reflecting the skin of the measured part was obtained with the following indicators: (I) Sa: the arithmetic average of the heights of each point within the area of the test; (II) Sq: the average root of the average square of the height of each point within the area of the test. The patients' satisfaction scores were assessed one month after the last treatment. The degree of satisfaction was divided into satisfaction with acne scar, satisfaction with large pores, satisfaction with safety, and calculated as: very satisfied $=2$; satisfied $=1$; no opinion $=0$; dissatisfied $=-1$; very dissatisfied $=-2$.

\section{Safety}

Immediate adverse effects (AEs), including erythema, edema, exudation, purpura, and pain, were recorded after each treatment. The visual scoring method assessed the pain $(0-10$ points, 0 points $=$ no pain, 10 points $=$ maximal pain). The mean scores of the four assessments were calculated. Long-term AEs, including hyperpigmentation, hypopigmentation, and pruritus, were evaluated one month after the last treatment. Duration of erythema, crust shedding time, and duration of pain were recorded.

\section{Statistical analysis}

SPSS software (version 22.0, IBM, Chicago, Illinois, UCA) was used for statistical analysis. Continuous variables were expressed as means \pm standard deviation (SD) and analyzed using the paired $t$-test. Categorial variables were presented as frequencies (percentage) and analyzed using the Chisquare test or Fisher's exact test. Two-tailed $\mathrm{P}<0.05$ was considered statistically significant.

\section{Results}

\section{Baseline characteristics of patients}

Twenty-four people were recruited for this study, two failed to complete all treatments, and the remaining 22 were included in the efficacy evaluation (Figure 1). Of the 22 patients, $4(18.2 \%)$ were male, with an average age of 29.68 years. The duration of acne scars was $8.8 \pm 3.4$ years. All patients had Fitzpatrick skin type IV. Other details are presented in Table 1 .

\section{Treatment efficacy}

Results of the ECCA assessment are shown in Table 2. Picosecond laser treatment significantly impacted all atrophic acne scars, with the notable difference before and after treatment $(\mathrm{P}=0.000$ for all types, $\mathrm{P}<0.001$ for $\mathrm{V}$-type, $\mathrm{P}=0.002$ for $\mathrm{U}$-type, and $\mathrm{P}=0.021$ for $\mathrm{M}$-type). Non-ablative fractional laser treatment resulted in a significant difference 


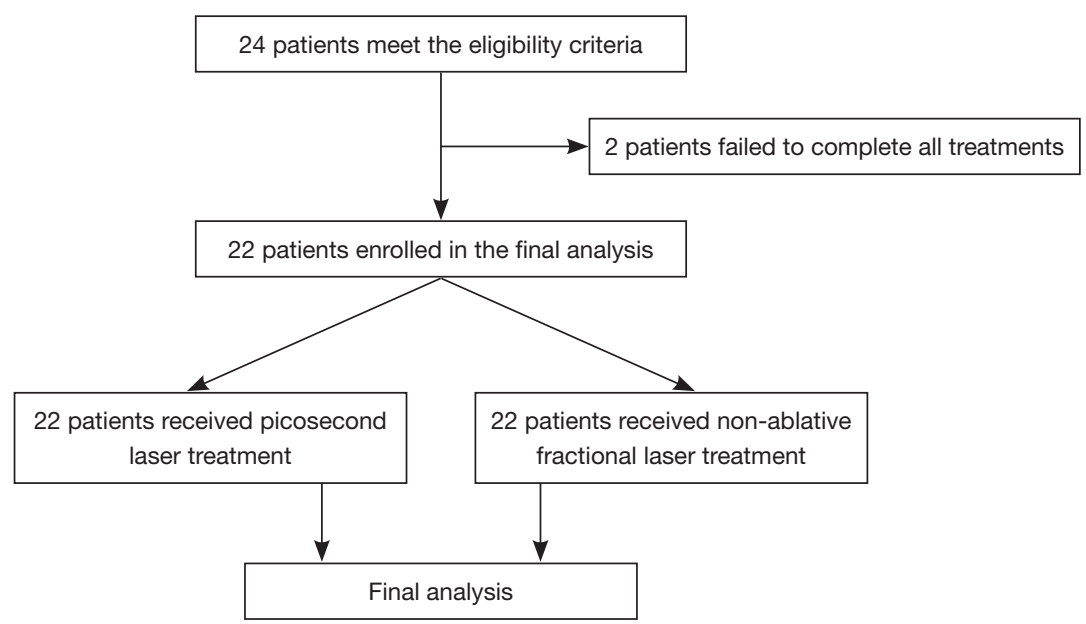

Figure 1 Flow diagram of participants enrollment.

Table 1 Baseline characteristics

\begin{tabular}{lc}
\hline Characteristics & $N(\%)$ or mean \pm SD \\
\hline Gender & $4(18.2)$ \\
Male & $18(81.8)$ \\
Female & $29.68 \pm 3.75$ \\
Age (years) & $8.8 \pm 3.4$ \\
Duration of acne scars (years) & \\
Side of picosecond laser & $13(59.1)$ \\
Left & $9(40.9)$ \\
Right & \\
Fitzpatrick skin type & 0 \\
III & $22(100.0)$ \\
IV &
\end{tabular}

Note: SD, standard deviation.

before and after treatment for V-type/M-type acne atrophic scars, but not for U-type. Compared with non-ablative fractional laser, the picosecond laser had a more pronounced effect on ECCA score for V-type and U-type acne atrophic scars, but not for M-type (see Figure 2 for a typical case).

The results of measuring the skin flatness with a threedimensional imaging system (PRIMOS) showed that the height of each treatment site after treatment and the variance of heights was significantly lower than that before treatment $(\mathrm{P}=0.041$ and $\mathrm{P}=0.02$ respectively) in the picosecond laser group but not in the non-ablative fractional laser group $(\mathrm{P}=0.785$ and $\mathrm{P}=0.519$ respectively).
Table 2 ECCA score

\begin{tabular}{lccc}
\hline ECCA score & $\begin{array}{c}\text { Picosecond } \\
\text { group }(\mathrm{n}=22)\end{array}$ & $\begin{array}{c}\text { Non-ablative } \\
\text { group }(\mathrm{n}=22)\end{array}$ & P value \\
\hline All type scar & & & \\
Before treatment & $21.67 \pm 3.22$ & $21.68 \pm 3.25$ & 0.658 \\
After treatment & $20.73 \pm 3.63$ & $21.25 \pm 3.26$ & 0.000 \\
P value & 0.000 & 0.001 & \\
V-type scar & & & \\
Before treatment & $17.90 \pm 0.30$ & $17.90 \pm 0.30$ & 1.000 \\
After treatment & $16.67 \pm 1.15$ & $17.52 \pm 0.68$ & 0.004 \\
P value & $<0.001$ & 0.017 & \\
U-type scar & & & \\
Before treatment & $21.47 \pm 0.81$ & $21.48 \pm 0.87$ & 1.000 \\
After treatment & $20.38 \pm 1.12$ & $21.00 \pm 1.00$ & 0.020 \\
P value & 0.002 & 0.096 & \\
M-type scar & & & 0.329 \\
Before treatment & $25.62 \pm 0.62$ & $25.67 \pm 0.73$ & \\
After treatment & $25.14 \pm 0.57$ & $25.24 \pm 0.44$ & 0.576 \\
P value & 0.021 & 0.047 & \\
\hline
\end{tabular}

Note: ECCA score, echelle d'evaluation clinique des cicatrices d'acne.

As shown in Table 3, compared to the Non-ablative fractional laser group, patients in the picosecond group were more satisfied with the appearance of skin pores (2.86 \pm 0.91 vs. $1.81 \pm 0.81, \mathrm{P}=0.001)$ and with the atrophic 

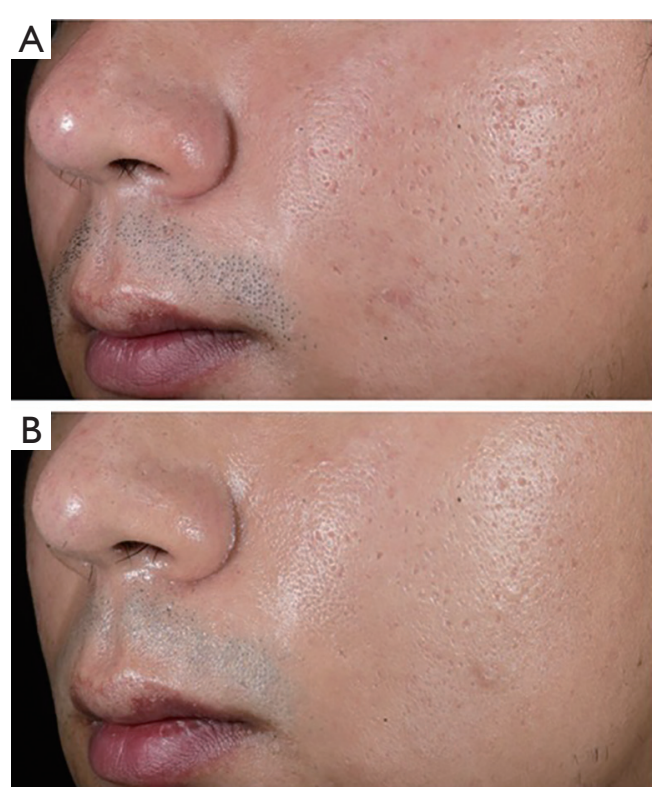

Figure 2 Effect of picosecond laser treatment on scars. (A) Before treatment, acne depressed scars, large pores and rough skin can be seen; (B) one month after four 532/1,064 nm picosecond laser treatments, the depressed scar of acne becomes shallow, the skin smoothness increases, and the pores shrink.

Table 3 Treatment, efficacy, and satisfaction

\begin{tabular}{lccc}
\hline Variables & $\begin{array}{c}\text { Picosecond } \\
\text { group }(n=22)\end{array}$ & $\begin{array}{c}\text { Non-ablative } \\
\text { group }(\mathrm{n}=22)\end{array}$ & P value \\
\hline PRIMOS score & & & \\
Sa before treatment & $66.99 \pm 35.46$ & $56.60 \pm 24.80$ & 0.316 \\
Sa after treatment & $48.96 \pm 20.85$ & $59.12 \pm 32.20$ & 0.132 \\
P value & 0.041 & 0.785 & \\
Sq before treatment $154.76 \pm 128.38$ & $123.90 \pm 96.80$ & 0.419 \\
Sq after treatment & $97.31 \pm 84.80$ & $117.16 \pm 108.00$ & 0.358 \\
P value & 0.020 & 0.519 & \\
$\begin{array}{l}\text { Patient satisfaction } \\
\text { Satisfaction with } \\
\text { pore }\end{array}$ & $2.86 \pm 0.91$ & $1.81 \pm 0.81$ & 0.001 \\
$\begin{array}{l}\text { Satisfaction with } \\
\text { acne scar }\end{array}$ & $2.93 \pm 0.88$ & $2.39 \pm 0.75$ & 0.009 \\
$\begin{array}{l}\text { Satisfaction with } \\
\text { safety }\end{array}$ & $3.55 \pm 0.70$ & $3.39 \pm 0.78$ & 0.137 \\
\hline
\end{tabular}

Note: PRIMOS, phaseshift rapid in vivo measurement of skin. $\mathrm{Sa}$, the arithmetic average of the heights of each point within the area of the test. Sq, the average root of the average square of the height of each point within the area of the test.
Table 4 Adverse effects

\begin{tabular}{lccc}
\hline AE & $\begin{array}{c}\text { Picosecond } \\
\text { group }(\mathrm{n}=22)\end{array}$ & $\begin{array}{c}\text { Non-exfoliation } \\
\text { group }(\mathrm{n}=22)\end{array}$ & P value \\
\hline Immediate AEs & & & \\
Erythema & $1.56 \pm 0.23$ & $1.44 \pm 0.24$ & 0.047 \\
Edema & $1.32 \pm 0.23$ & $1.27 \pm 0.25$ & 0.383 \\
Exudation & $0.30 \pm 0.22$ & $0.33 \pm 0.38$ & 0.751 \\
$\begin{array}{l}\text { Purpura } \\
\text { Pain }\end{array}$ & $0.57 \pm 0.40$ & $0.55 \pm 0.42$ & 0.888 \\
Long-term AEs & $4.19 \pm 1.79$ & $4.75 \pm 1.63$ & 0.616 \\
$\begin{array}{l}\text { Duration of } \\
\text { erythema }\end{array}$ & $3.24 \pm 2.15$ & $3.29 \pm 3.85$ & 0.929 \\
Crust shedding time & $2.73 \pm 0.87$ & $3.12 \pm 1.44$ & 0.517 \\
Hyperpigmentation & $0.13 \pm 0.35$ & $0.17 \pm 0.43$ & 0.590 \\
Hypopigmentation & $0.50 \pm 1.12$ & $0.51 \pm 1.24$ & 0.803 \\
Pruritus & $0.61 \pm 1.50$ & $0.46 \pm 1.15$ & 0.143 \\
Duration of pain & $1.35 \pm 3.17$ & $1.43 \pm 3.36$ & 0.192 \\
\hline
\end{tabular}

Note: AE, adverse event.

scars $(2.93 \pm 0.88$ vs. $2.39 \pm 0.75, \mathrm{P}=0.009)$. There was no difference in the satisfaction with safety between the two groups $(\mathrm{P}=0.137)$.

\section{Treatment safety}

As shown in Table 4, among immediate responses, the average value of point pain was higher in the non-ablative group, while the erythema occurrence rate was slightly higher in the picosecond group. There was no significant difference noted in the occurrence of edema, exudation, or purpura between the groups (all $\mathrm{P}>0.05$ ).

For the long-term AEs, prolonged erythema duration and pigmentation were noted more often in the nonablative group, while itching was more common in the picosecond group, but without significant difference (all $\mathrm{P}>0.05)$.

\section{Discussion}

The application of picosecond lasers in the treatment of acne scars is not uncommon, but quality evidence on its efficacy is still lacking (4). Among recently published studies on laser therapy, there were only 11 studies on picosecond 
lasers involving a cumulative total of 194 subjects (8). Thus, our study aimed to systemically compare the efficacy and safety of picosecond laser and non-ablative fractional laser in treating acne atrophic scars.

We used a universal ECCA score to assess the acne scar changes one month after the last treatment and the measurements of treatment site height by a rapid skin threedimensional imaging system to ensure the reproducibility of results. Compared to previous studies, based mostly on clinical evaluation, a professional PRIMOS instrument for evaluating skin smoothness was used, reflecting lasers' effect on skin's smoothness more objectively. This study was the first to report the improvement of acne scars and large pores in type IV skin in an Asian population to the best of our knowledge. In this study, picosecond treatment parameters are thus worth popularizing in the clinical application for skin types III-IV. It was found that Picosecond laser treatment had a significant impact on all types of atrophic acne scars, with the notable difference before and after treatment, while non-ablative laser treatment resulted in a significant difference for $\mathrm{V}$-type/M-type acne atrophic scars, but not for U-type. The results of measuring the skin flatness with a non-invasive PRIMOS system showed that each treatment site's height after treatment and variance of heights were significantly lower than before treatment in the picosecond group, but not in the non-ablative group. In contrast, the reported erythema rate was slightly higher in patients treated with Picosecond laser, while edema, exudation, purpura, pain, and long-term AEs were similar between the groups.

The picosecond laser was previously used to improve photoaging, and its effect was widely recognized $(4-6,9)$. However, whether a picosecond laser can treat acne atrophic scars was still under discussion in China and abroad. A recently published study by Manuskiatti et al. (10) reported long-term results of applying 1064-nm picosecond laser in acne scar treatment. A study by Dr. Manuskiatti enrolled 26 patients of Asian origin and Thai nationality, with the skin types like those in our study, and $50 \%$ of the subjects were rated as having scar improvement not less than $50 \%$. Interestingly, discussing adverse effects, the authors noted the higher rate of post-inflammatory hyperpigmentation, along with more commonly described erythema. Although, being more comparative by design, our study reported smaller incidence of erythema and hyperpigmentation using the same type of laser, and those results are closer to recent studies published in China $(11,12)$ and Korea (13), which may be explained by differences in procedure duration, size of the treatment area or post-treatment management. In any case, post-inflammatory hyperpigmentation in dark skin types after application of picosecond laser calls to be closer investigated in later studies.

We hypothesized that picosecond laser could improve acne atrophic scars, and the observed effect was better than that of non-ablative fractional laser. Concurrently, the picosecond laser can significantly improve the large pores and gloss of skin, as noted both by patients and doctors. Zhang et al. (12) study is the most recent report on picosecond laser usage in Chinese patients, dated 2019. The authors used a picosecond alexandrite laser with a diffractive lens array and reported this technique to lessen the procedure's pain. Although there was no statistical difference in pain scores between non-ablative fractional laser group and the picosecond group in our study, we also noted the same trend in the picosecond group compared to non-ablative $(4.19 \pm 1.79$ vs. $4.75 \pm 1.63)$. Considering that this study's sample size is 22 (however, which is relatively close to the sample size of similar studies), perhaps the sample size is too small to find the statistical difference. Future studies with larger sample sizes could verify whether applying a picosecond laser truly reduces the pain burden.

As for the safety of the procedure, all recent studies reported picosecond laser (Nd: YAG) showed comparable or better safety profile compared to non-ablative $1,550-\mathrm{nm}$ erbium-glass laser $(13,14)$, fractional 1,550-nm erbium fiber laser (15), and fractional carbon dioxide laser (16). The study by Chayavichitsilp et al. (15), comparing Nd: YAG 1,064-nm picosecond laser and fractional 1,550-nm erbium fiber laser, reported that while there was no significant difference between 1,064-nm picosecond laser group and fractional 1,550-nm erbium fiber laser group in terms of median ECCA score improvement, more pinpoint bleeding (petechiae) was significantly observed with the picosecond laser, while more pain was noted with the erbium laser. Bernstein et al. (9) in 2017 included 27 patients with acne scarring and used dual-wavelength 1,064 and $532 \mathrm{~nm}$ picosecond-domain lasers and reported petechiae among adverse effects; however, there was a higher frequency of post-treatment erythema and edema. Other studies, exploring the safety of 532 and 1,064 nm picosecond laser in dermatology, reported erythema being the most frequent and sometimes only one noted complication in patients treated for facial photodamage (9), hyperpigmentation (16), solar lentigines (17), melasma (18), striae alba (19) and management of wrinkles $(20,21)$ or verrucous epidermal nevi (22). The occurrence of erythema-like changes as the 
most frequent complication following the picosecond laser application was noted in our study. The fact may explain that the picosecond laser penetrates more deeply into the dermis (23) and shows a greater extent of laser-induced damage, activating inflammatory cytokine signaling (7). According to other reports, those changes are transient, not related to the pulse frequency (24), and last up to 6 (in rare cases 12$)$ weeks $(8,25)$.

Along with erythema, the next most often noted adverse effect in our study was edema: at once after treatment, reported edema was slightly heavier in the picosecond group than in the non-ablative group, but there was no difference in the other immediate and long-term adverse reactions between the two groups. No new adverse reactions were found, showing that the $1,064 / 532 \mathrm{~nm}$ picosecond laser therapy has satisfactory safety. Conversely, Wen et al. (26) in the recent study showed some efficacy of picosecond Alexandrite laser in treating post-inflammatory erythema, implying that the LIOB effect may be used in erythema reduction and in stimulating collagen production.

Our study has some limitations. First, the study only included patients with Fitzpatrick skin type IV. Patients with other skin types and laser treatment with different energy should be assessed in future studies. Second, although comparable to other similar studies, the number of patients is still too low for the full statistical analysis. And finally, the follow-up duration was 6 months, but it could be lengthened to obtain longer-term results.

In conclusion, Fractionated frequency-doubled 1,064/532 nm Picosecond Nd: YAG laser showed better efficacy in treating acne atrophic scars than the alternative and satisfactory safety and added improvement of the large pores and gloss of skin. Medical laser has been widely used in the treatment for facial atrophic scars in recent years but with insufficient clinical evidence. In the future, more randomized controlled trials should be carried out to get strong evidence for the reasonable application of laser in the treatment of scar.

\section{Acknowledgments}

Funding: Project fund of Shanghai Municipal Health Commission (201840236).

\section{Footnote}

Trial Protocol: Available at http://dx.doi.org/10.21037/atm21-1715
Reporting Checklist: The authors have completed the CONSORT reporting checklist. Available at http://dx.doi. org/10.21037/atm-21-1715

Data Sharing Statement: Available at http://dx.doi. org/10.21037/atm-21-1715

Conflicts of Interest: All authors have completed the ICMJE uniform disclosure form (available at http://dx.doi. org/10.21037/atm-21-1715). The authors have no conflicts of interest to declare.

Ethical Statement: The authors are accountable for all aspects of the work in ensuring that questions related to the accuracy or integrity of any part of the work are appropriately investigated and resolved. The study was performed following the Declaration of Helsinki (as revised in 2013) principles and approved by the Ethics Committee of Tongji University Affiliated Shanghai Skin Disease Hospital (Approval number: 2020-20). All the patients signed written informed consent.

Open Access Statement: This is an Open Access article distributed in accordance with the Creative Commons Attribution-NonCommercial-NoDerivs 4.0 International License (CC BY-NC-ND 4.0), which permits the noncommercial replication and distribution of the article with the strict proviso that no changes or edits are made and the original work is properly cited (including links to both the formal publication through the relevant DOI and the license). See: https://creativecommons.org/licenses/by-nc-nd/4.0/.

\section{References}

1. Rao J. Treatment of acne scarring. Facial Plast Surg Clin North Am 2011;19:275-91.

2. Ip A, Muller I, Geraghty AWA, et al. Views and experiences of people with acne vulgaris and healthcare professionals about treatments: systematic review and thematic synthesis of qualitative research. BMJ Open 2021;11:e041794.

3. Abdel Hay R, Shalaby K, Zaher H, et al. Interventions for acne scars. Cochrane Database Syst Rev 2016;4:CD011946.

4. Barbaric J, Abbott R, Posadzki P, et al. Light therapies for acne. Cochrane Database Syst Rev 2016;9:CD007917.

5. Sobanko JF, Alster TS. Management of acne scarring, part I: a comparative review of laser surgical approaches. Am J 
Clin Dermatol 2012;13:319-30.

6. Sadick NS, Cardona A. Laser treatment for facial acne scars: A review. J Cosmet Laser Ther 2018;20:424-35.

7. Bernstein EF, Schomacker KT, Basilavecchio LD, et al. A novel dual-wavelength, Nd:YAG, picosecond-domain laser safely and effectively removes multicolor tattoos. Lasers Surg Med 2015;47:542-8.

8. Wu DC, Goldman MP, Wat H, et al. A Systematic Review of Picosecond Laser in Dermatology: Evidence and Recommendations. Lasers Surg Med 2021;53:9-49.

9. Bernstein EF, Schomacker KT, Basilavecchio LD, et al. Treatment of acne scarring with a novel fractionated, dual-wavelength, picosecond-domain laser incorporating a novel holographic beam-splitter. Lasers Surg Med 2017;49:796-802.

10. Manuskiatti W, Punyaratabandhu P, Tantrapornpong $\mathrm{P}$, et al. Objective and Long-Term Evaluation of the Efficacy and Safety of a 1064-nm Picosecond Laser With Fractionated Microlens Array for the Treatment of Atrophic Acne Scar in Asians. Lasers Surg Med 2020. [Epub ahead of print]. doi: 10.1002/1sm.23368.

11. Yang CS, Huang YL, Cheng CY, et al. A Prospective Study of Fractionated Dual-Wavelength Picosecond Laser in Treating Acne Scar. Lasers Surg Med 2020;52:735-42.

12. Zhang M, Fang J, Wu Q, et al. Evaluation of the Safety and Efficacy of a Picosecond Alexandrite Laser With DLA for Acne Scars in Chinese Patients. Lasers Surg Med 2020;52:176-81.

13. Kwon HH, Yang SH, Cho YJ, et al. Comparison of a 1064-nm neodymium-doped yttrium aluminum garnet picosecond laser using a diffractive optical element vs. a nonablative 1550 -nm erbium-glass laser for the treatment of facial acne scarring in Asian patients: a 17-week prospective, randomized, split-face, controlled trial. J Eur Acad Dermatol Venereol 2020;34:2907-13.

14. Zaleski-Larsen LA, Jones IT, Guiha I, et al. A Comparison Study of the Nonablative Fractional 1565-nm Er: glass and the Picosecond Fractional 1064/532-nm Nd: YAG Lasers in the Treatment of Striae Alba: A Split Body DoubleBlinded Trial. Dermatol Surg 2018;44:1311-6.

15. Chayavichitsilp P, Limtong $P$, Triyangkulsri K, et al. Comparison of fractional neodymium-doped yttrium aluminum garnet (Nd:YAG) 1064-nm picosecond laser and fractional 1550-nm erbium fiber laser in facial acne scar treatment. Lasers Med Sci 2020;35:695-700.

16. Sirithanabadeekul P, Tantrapornpong P, Rattakul B, et al. Comparison of Fractional Picosecond 1064-nm Laser and Fractional Carbon Dioxide Laser for Treating Atrophic
Acne Scars: A Randomized Split-Face Trial. Dermatol Surg 2021;47:e58-65.

17. Artzi O, Mehrabi JN, Koren A, et al. Picosecond 532-nm neodymium-doped yttrium aluminium garnet laser-a novel and promising modality for the treatment of café-au-lait macules. Lasers Med Sci 2018;33:693-7.

18. Guss L, Goldman MP, Wu DC. Picosecond 532 nm Neodymium-Doped Yttrium Aluminium Garnet Laser for the Treatment of Solar Lentigines in Darker Skin Types: Safety and Efficacy. Dermatol Surg 2017;43:456-9.

19. Chalermchai T, Rummaneethorn P. Effects of a fractional picosecond $1,064 \mathrm{~nm}$ laser for the treatment of dermal and mixed type melasma. J Cosmet Laser Ther 2018;20:134-9.

20. Kirsanova L, Araviiskaia E, Rybakova M, et al. Histological characterization of age-related skin changes following the use of picosecond laser: Low vs high energy. Dermatol Ther 2020;33:e13635.

21. Saluja R, Gentile RD. Picosecond Laser: Tattoos and Skin Rejuvenation. Facial Plast Surg Clin North Am 2020;28:87-100.

22. Levi A, Amitai DB, Mimouni D, et al. Picosecond 532nm neodymium-doped yttrium aluminum garnet laser-a promising modality for the management of verrucous epidermal nevi. Lasers Med Sci 2018;33:597-601.

23. Gold MH. Dual wavelength treatment protocol with a picosecond laser for the reduction of facial wrinkles. J Cosmet Laser Ther 2019;21:147-51.

24. Dierickx C. Using normal and high pulse coverage with picosecond laser treatment of wrinkles and acne scarring: Long term clinical observations. Lasers Surg Med 2018;50:51-5.

25. Ohshiro T, Ohshiro T, Sasaki K, et al. Picosecond pulse duration laser treatment for dermal melanocytosis in Asians: A retrospective review. Laser Ther 2016;25:99-104.

26. Wen X, Li Y, Hamblin MR, Jiang X. A randomized split-face, investigator-blinded study of a picosecond Alexandrite laser for post-inflammatory erythema and acne scars. Dermatol Ther 2020;33:e13941.

(English Language Editor: J. Chapnick)

Cite this article as: Shi Y, Jiang W, Li W, Zhang W, Zou Y. Comparison of fractionated frequency-doubled 1,064/532 nm picosecond Nd:YAG lasers and non-ablative fractional 1,540 nm Er: glass in the treatment of facial atrophic scars: a randomized, split-face, double-blind trial. Ann Transl Med 2021;9(10):862. doi: 10.21037/atm-21-1715 